\title{
Lack of EC-SOD worsens alveolar and vascular development in a neonatal mouse model of bleomycin-induced bronchopulmonary dysplasia and pulmonary hypertension
}

\author{
Cassidy Delaney ${ }^{1}$, Rachel H. Wright' ${ }^{1}$, Jen-Ruey Tang ${ }^{1}$, Crystal Woods', Leah Villegas ${ }^{1}$, Laurie Sherlock', Rashmin C. Savani ${ }^{2}$, \\ Steven H. Abman' and Eva Nozik-Grayck'
}

BACKGROUND: Pulmonary hypertension (PH) worsens clinical outcomes in former preterm infants with bronchopulmonary dysplasia (BPD). Oxidant stress disrupts alveolar and vascular development in models of BPD. Bleomycin causes oxidative stress and induces BPD and PAH in neonatal rats. Disruption in the vascular endothelial growth factor (VEGF) and nitric oxide signaling pathways contributes to BPD. We hypothesized that loss of EC-SOD would worsen PAH associated with BPD in a neonatal mouse model of bleomycin-induced BPD by disrupting the VEGF/NO signaling pathway.

METHODS: Neonatal wild-type mice (WT), and mice lacking EC-SOD (EC-SOD KO) received intraperitoneal bleomycin (2 units $/ \mathrm{kg}$ ) or phosphate-buffered saline (PBS) three times weekly and were evaluated at weeks 3 or 4 .

RESULTS: Lack of EC-SOD impaired alveolar development and resulted in $\mathrm{PH}$ (elevated right ventricular systolic pressures, right ventricular hypertrophy (RVH)), decreased vessel density, and increased small vessel muscularization. Exposure to bleomycin further impaired alveolar development, worsened RVH and vascular remodeling. Lack of EC-SOD and bleomycin treatment decreased lung total and phosphorylated VEGFR2 and eNOS protein expression.

CONCLUSION: EC-SOD is critical in preserving normal lung development and loss of EC-SOD results in disrupted alveolar development, PAH and vascular remodeling at baseline, which is further worsened with bleomycin and associated with decreased activation of VEGFR2.

B ronchopulmonary dysplasia (BPD) is a common chronic respiratory disease following preterm birth in infants who were mechanically ventilated or treated with supplemental oxygen. The clinical course of infants with BPD may be complicated by sustained oxygen dependency, prolonged need for ventilator support, frequent hospitalizations, and pulmonary hypertension (PH). It is estimated that $\mathrm{PH}$ develops in 14$25 \%$ of preterm infants with $\mathrm{BPD}(1,2)$ and is associated with high mortality (30-48\%) (3). Despite advances in neonatal medicine, development of BPD complicated by $\mathrm{PH}$ remains a significant cause of long-term morbidity and mortality in this patient population, and a better understanding of disease pathogenesis is required to improve treatment strategies.

The immature lung is susceptible to oxidative stress due to insufficient antioxidant defenses, which contributes to the pathogenesis of BPD and $\mathrm{PH}(4,5)$. One key antioxidant enzyme, extracellular superoxide dismutase (EC-SOD) is highly expressed in the lung and vasculature and is tightly regulated in the developing lung (6). This antioxidant is one of the three mammalian isoforms of the superoxide dismutases (SOD), which catalyze the dismutation of superoxide to hydrogen peroxide and oxygen (6). Lung EC-SOD expression and activity is impaired in a number of models of BPD and PH, and the level of EC-SOD expression modulates severity of lung and vascular disease in adult and neonatal animal models, including in hyperoxia-induced BPD, chronic hypoxic $\mathrm{PH}$, and bleomycin-induced lung fibrosis (4,7-9). While neonatal mice overexpressing EC-SOD are protected from BPD seen with chronic hyperoxia, the impact of the loss of EC-SOD on alveolar development, $\mathrm{PH}$, and vascular growth in the newborn lung is not well understood (4). Collectively, these studies provide a strong rationale to further examine the role of EC-SOD in development of BPD complicated by PH.

One robust neonatal rat model of BPD and PH employs intraperitoneal administration of bleomycin to augment the inflammatory response leading to impaired alveolar and vascular growth (10). Bleomycin, a chemotherapeutic agent, produces significant oxidative stress and inflammation $(11,12)$. It is well established that bleomycin causes lung fibrosis in the mature lung of rodents and humans, but in contrast, systemic administration of bleomycin to neonatal rats results in BPD and PH, evidenced by impaired alveolar and vascular development, increased pulmonary vascular resistance and right ventricular failure $(4,9,10,13,14)$. Our study adapted the bleomycin model to mice to enable us to test the hypothesis that loss of EC-SOD would worsen PH associated with BPD in a neonatal mouse model of bleomycin-induced BPD by 
disrupting key signaling pathways. We focused on the vascular endothelial growth factor (VEGF)/VEGFR2/nitric oxide (NO) pathway, which is critical to postnatal alveolar and vascular development (15-18).

\section{RESULTS}

Neonatal Mice Lacking EC-SOD Demonstrate Disrupted Alveolar Development at Baseline and an Exaggerated Response to Treatment With Bleomycin

Alveolar development was evaluated in 4-wk-old WT and EC-SOD KO mice born and raised at Denver altitude and treated in the neonatal period with IP phosphate-buffered saline (PBS) or bleomycin. A representative image of a pentachrome stained lung for each group illustrates the differences in alveolar development (Figure 1a-d). Alveolar structure was quantified by morphometric analysis of the radial alveolar
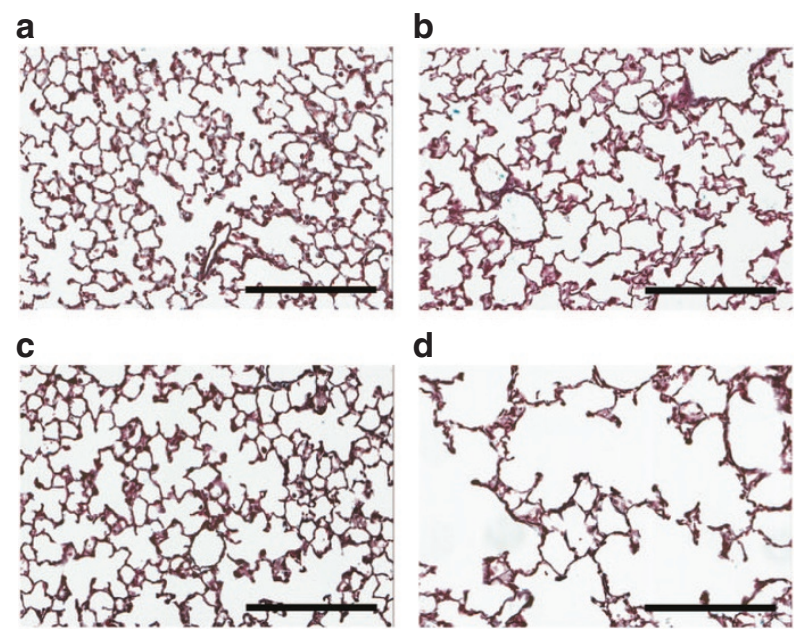

d
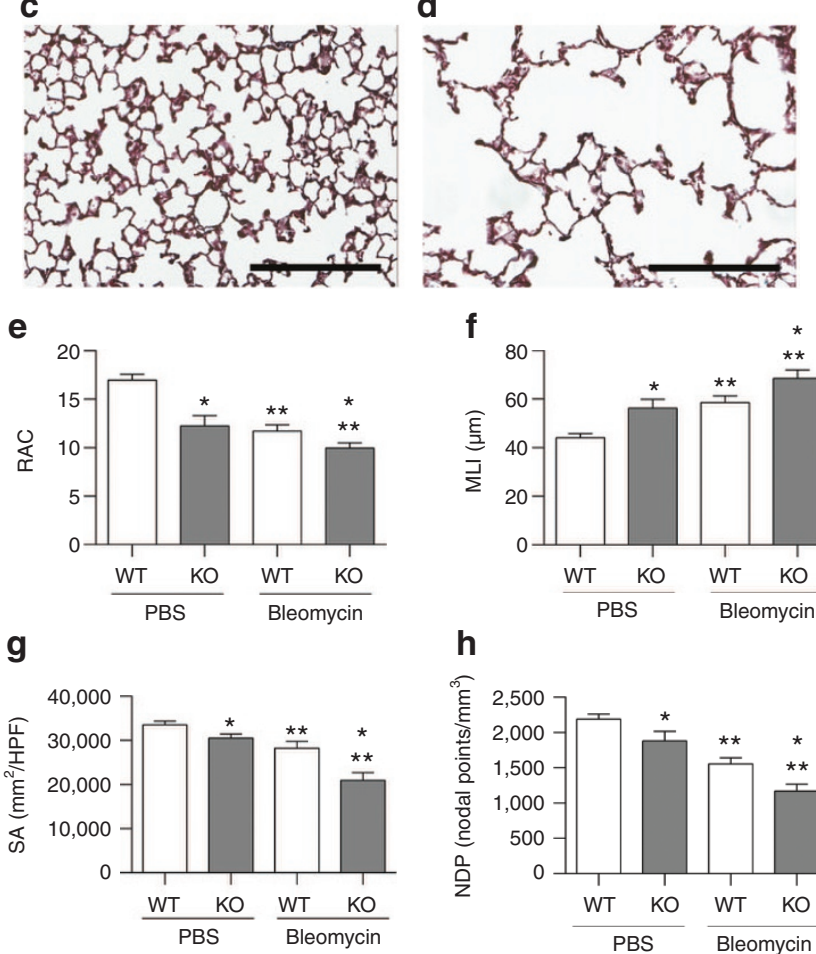

f

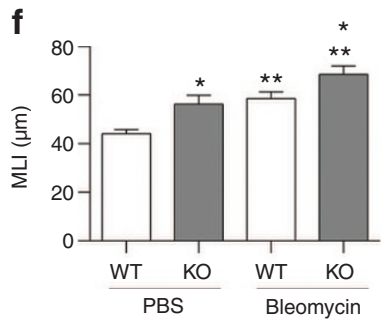

h

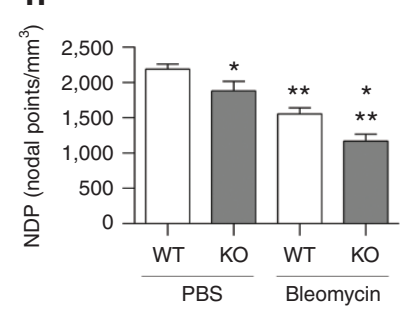

Figure 1. Loss of EC-SOD impairs alveolar development at baseline and worsens bleomycin-induced BPD. (a-d) Representative image of pentachrome stain of lung sections from 4-wk-old WT and EC-SOD $\mathrm{KO}$ mice exposed to IP phosphate-buffered saline (PBS) or bleomycin. (a) WT PBS, (b) WT Bleo, (c) KO PBS, (d) KO Bleo, scale bar = 200 microns. (e-h) Morphometric analysis, radial alveolar counts, mean linear intercept, nodal point density, and surface area of WT and EC-SOD KO mice exposed to IP PBS or Bleomycin. ${ }^{*} P<0.05$ for strain, ${ }^{* *} P<0.01$ for treatment by two-way ANOVA, $n=4-9$.

counts, mean linear intercept, nodal point density, and surface area (Figure 1e-h). We measured an overall significant decrease in alveolar development in the EC-SOD KO mice compared to the WT mice by two-way ANOVA for radial alveolar counts $\left({ }^{\star} P<0.05\right)$, mean linear intercept $\left({ }^{\star} P<0.05\right)$, nodal point density $\left({ }^{\star} P<0.01\right)$, and surface area $\left({ }^{\star} P<0.01\right)$. Furthermore, we detected a significant impairment in alveolar development in response to bleomycin in both strains by two-way ANOVA for radial alveolar counts $\left({ }^{*} P<0.001\right)$, mean linear intercept $\left({ }^{* *} P<0.001\right)$, nodal point density $\left({ }^{*} P\right.$ $<0.001)$, and surface area $\left({ }^{*} P<0.0001\right)$. The EC-SOD KO mouse exposed to bleomycin consistently showed the greatest impairment by all four parameters.

\section{Mice Lacking EC-SOD Exhibited Pulmonary Hypertension at Baseline, Which Was Worsened by Bleomycin}

$\mathrm{PH}$ was first evaluated in $4 \mathrm{wk}$ mice, at the same time point in which alveolar development was assessed. At $4 \mathrm{wk}$, the PBStreated EC-SOD mice showed an increase in $\mathrm{RV} / \mathrm{LV}+\mathrm{S}$ weight and an increase in right ventricular systolic pressures compared to PBS-treated WT mice (Figure 2a,b, $P<0.05$ ). After bleomycin treatment, however, neither strain showed an increase in $\mathrm{RV} / \mathrm{LV}+\mathrm{S}$ compared to PBS-treated WT mice, and the right ventricular systolic pressure was similar to baseline measurements for each strain (Figure 2a,b). To evaluate whether the lack of change in $\mathrm{RV} / \mathrm{LV}+\mathrm{S}$ with bleomycin could be due to left ventricular enlargement, we analyzed the left ventricle weights, and observed that both strains showed an increase in LV/body weight, following bleomycin (Figure 2c, $P<0.05$ ). We then a

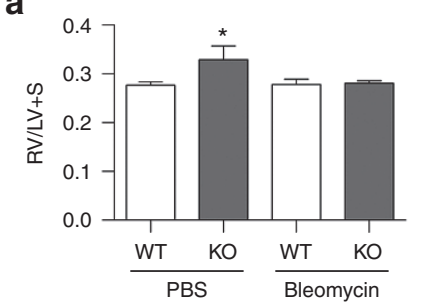

C

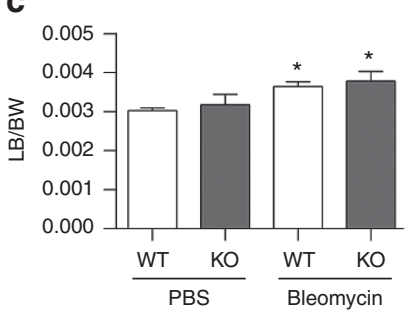

b

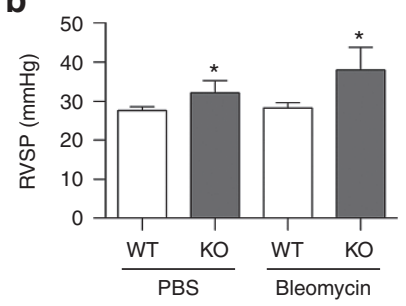

d

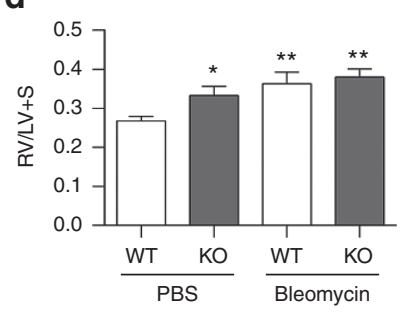

Figure 2. Loss of EC-SOD causes pulmonary hypertension at baseline in neonatal mice, which is worsened by treatment with bleomycin. (a) RV/ $\mathrm{LV}+\mathrm{S}$ weight at $4 \mathrm{wk}$ in WT and EC-SOD KO mice following IP phosphatebuffered saline (PBS) or bleomycin treatment, ${ }^{*} P<0.05$ for strain by twoway ANOVA. (b) Right ventricular systolic pressures by direct RV puncture at 4 wk of age in WT and EC-SOD KO mice following IP PBS or bleomycin treatment, ${ }^{*} P<0.01$ for strain by two-way ANOVA. (c) LV/body weight at $4 \mathrm{wk}$ of age in WT and EC-SOD KO mice following IP PBS or bleomycin, ${ }^{*} P<0.05$ for treatment by two-way ANOVA. (d) RV/LV+S weight at 3 wk in WT and EC-SOD KO mice following IP PBS or bleomycin treatment, ${ }^{*} P=0.06$ for strain, ${ }^{* *} P<0.005$ for treatment by two-way ANOVA, $n=3-8$. 


\section{Articles | Delaneyetal.}

evaluated RVH at $3 \mathrm{wk}$, which was the time point following the completion of bleomycin injections, prior to any potential recovery from bleomycin. At $3 \mathrm{wk}$, EC-SOD KO mice tended to have an increase in $\mathrm{RV} / \mathrm{LV}+\mathrm{S}$ compared to WT mice (Figure $2 \mathrm{~d},{ }^{*} P=0.06$ ); bleomycin treatment caused an increase in $\mathrm{RV} /$ $\mathrm{LV}+\mathrm{S}$ in both the WT and EC-SOD KO bleomycin-treated mice developed RVH (Figure 2d, ${ }^{*} P<0.005$ ). Bleomycin did not change $\mathrm{LV}$ weight at the 3 -wk time point (data not shown).

\section{Pulmonary Vascular Structure Is Abnormal in Neonatal Mice Lacking EC-SOD and Treatment With Bleomycin Further Impairs Vascular Development}

We detected a decrease in vessel density and an increase in the percent of muscularized small vessels in KO mice at baseline and following treatment with bleomycin. We counted the number of small vessels ( $<30$ microns) to assess vessel density, as an indicator of vascular development. Vessel density tended to decrease at baseline in EC-SOD KO animals treated with PBS compared to WT PBS-treated animals $\left({ }^{*} P=\right.$ 0.1 . Treatment with bleomycin significantly decreased vessel density in both neonatal WT and EC-SOD KO mice, with no strain differences $\left({ }^{*} P<0.0001\right.$, Figure $\left.3 \mathrm{a}-\mathrm{e}\right)$. In addition, the ratio of muscularized small vessels/total number of small vessels increased in the PBS-treated EC-SOD KO mice compared to the WT mice, $\left({ }^{\star} P<0.0001\right.$, Figure $\left.4 a-e\right)$. Furthermore,

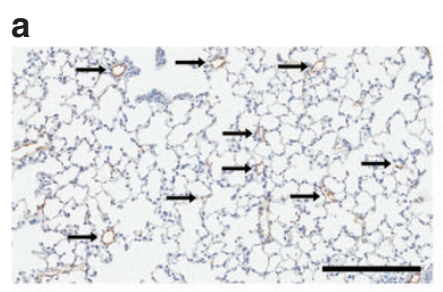

b

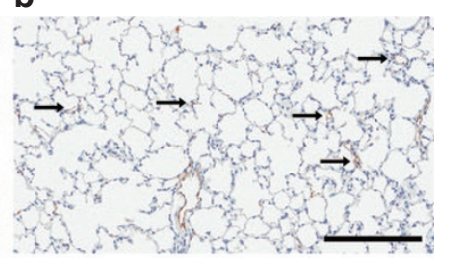

c

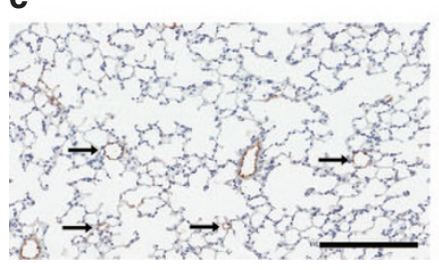

d
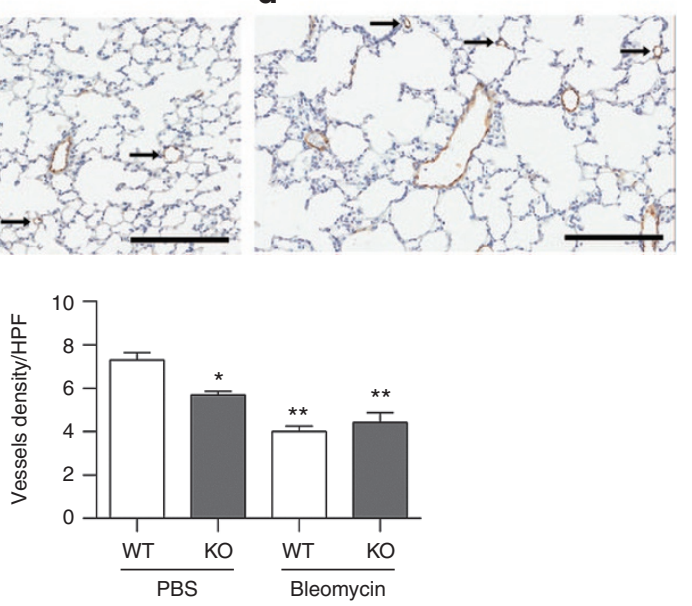

Figure 3. Pulmonary vascular density is decreased in neonatal mice lacking EC-SOD. Treatment with bleomycin further decreases vessel density. (a-d) Representative factor VIII staining in 4-wk-old WT and EC-SOD KO mice treated with IP PBS or bleomycin, (a) WT PBS, (b) WT Bleo, (c) KO PBS, (d) $\mathrm{KO}$ Bleo, arrows indicate vessels $<30$ microns, scale bar $=200$ microns. (e) Vessel density in WT and EC-SOD KO mice following IP PBS or bleomycin treatment, ${ }^{*} P=0.1$ for strain, ${ }^{* *} P<0.0001$ for treatment by two-way ANOVA, $n=3-5$. bleomycin increased the ratio of muscularized/total vessels in both strains, with a greater change in EC-SOD KO compared to WT mice $\left({ }^{* *} P<0.0001\right.$, Figure $\left.4 a-e\right)$.

\section{Lack of EC-SOD and Treatment With Bleomycin Decrease Pulmonary Expression of VEGFR2 and eNOS}

We evaluated lung VEGF, VEGFR2, and eNOS expression because this pathway is critically important in the regulation of normal alveolar and vascular development. Decreased signaling through this pathway has been associated with the development of BPD and PH in experimental models and preterm infants. At $3 \mathrm{wk}$, we detected a decrease between strains at baseline in the protein expression of the VEGF receptor, VEGFR2; and the active phosphorylated form of the receptor, pVEGFR2. We found a 58\% loss of lung VEGFR2 expression in mice lacking EC-SOD at baseline, $\left({ }^{\star} P<0.05\right.$, Figure $\left.5 \mathbf{b}\right)$ and a decrease in active pVEGFR2 with bleomycin treatment, $\left({ }^{\star} P<0.01\right.$, Figure $\left.5 c\right)$. VEGF regulates pulmonary vascular development in the neonatal lung in part via induction of eNOS and NO-dependent signaling; therefore, we measured eNOS expression in whole lung homogenates. EC-SOD KO mice tended to have a decrease in eNOS expression at baseline, $\left({ }^{\star} P<0.06\right.$, Figure $\left.5 d\right)$. Treatment with bleomycin resulted in a significant decrease in eNOS expression in WT animals $\left({ }^{*} P\right.$ $<0.0001$, Figure 5d). At 3 wk of age, we detected no difference a

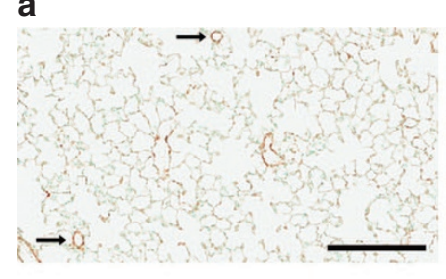

c

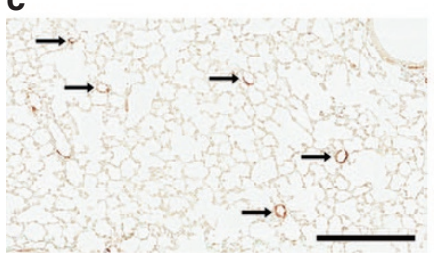

e

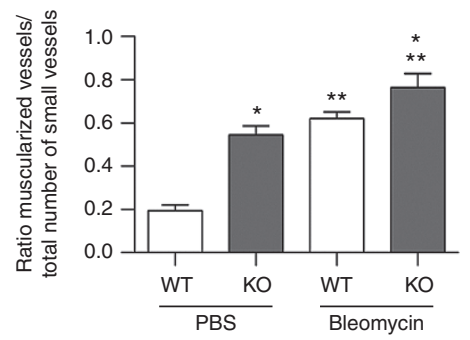

b

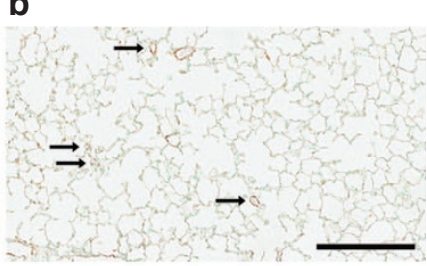

d

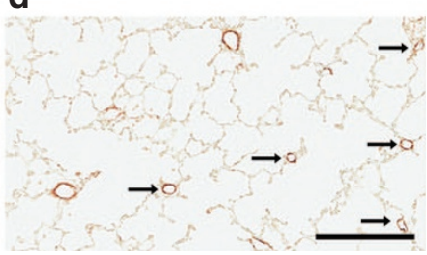




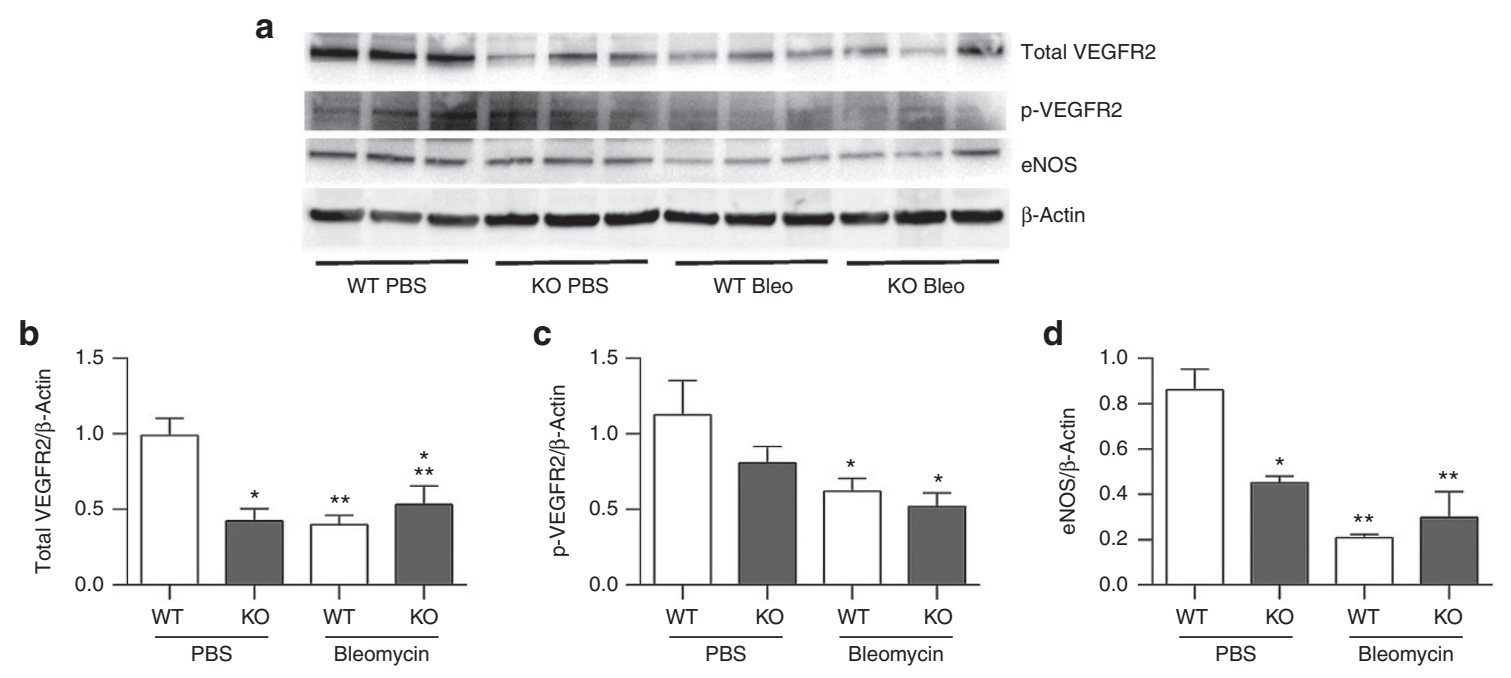

Figure 5. Lack of EC-SOD and treatment with bleomycin decrease pulmonary expression of total and active VEGRFR2 and eNOS. (a) Western blot analysis for total VEGFR2, pVEGFR2, eNOS, and $\beta$-actin. (b) Analysis of protein expression for total VEGFR2 standardized to $\beta$-actin, ${ }^{*} P<0.05$ for strain and ${ }^{* *} P<0.05$ for treatment by two-way ANOVA. (c) Active phosphorylated VEGFR2 relative to $\beta$-actin, ${ }^{*} P<0.01$ for treatment by two-way ANOVA. (d) eNOS expression relative to $\beta$-actin, ${ }^{*} P<0.06$ for strain, ${ }^{* *} P<0.0001$ for treatment by two-way ANOVA, $n=3-6$.

in the expression of VEGF and by $4 \mathrm{wk}$, there were no longer changes in the expression pattern of eNOS or VEGFR2 (not shown).

\section{Bleomycin Treatment Did Not Alter Lung EC-SOD Expression or Activity}

We did not observe a change in lung EC-SOD protein expression (Figure 6a,b) or activity (Figure 6c) in WT mice $3 \mathrm{wk}$ after treatment with bleomycin compared to control mice.

\section{DISCUSSION}

In this study, we hypothesized that loss of EC-SOD would worsen $\mathrm{PH}$ associated with BPD in a neonatal mouse model of bleomycin-induced BPD by disrupting the VEGF/VEGFR2/ NO pathway. We tested this hypothesis in neonatal mice lacking EC-SOD compared to WT control mice. We demonstrated that EC-SOD KO mice exhibit disrupted alveolar development, $\mathrm{PH}$ and pulmonary vascular remodeling at baseline; in a bleomycin model of BPD and PH mice lacking EC-SOD displayed more severe alveolar simplification, $\mathrm{PH}$, and disrupted pulmonary vascularization compared to WT mice; and the augmented injury was associated with significant decreases in the VEGF/VEGFR2/NO pathway. These data demonstrate the key role for EC-SOD in neonatal alveolar and vascular development and response to neonatal injury.

The first novel finding in this study is that mice lacking EC-SOD have abnormal lung development at baseline and worsened BPD in response to neonatal treatment with bleomycin. This is the first detailed morphometric analysis of alveolar development in immature EC-SOD KO mice, although this strain has been used in numerous adult mouse studies without any description of abnormal lung structure under control conditions $(15,19)$. It is possible that the impaired alveolar development in EC-SOD KO mice is due to a slower rate of alveolar growth that is no longer apparent in fully mature mice, or that

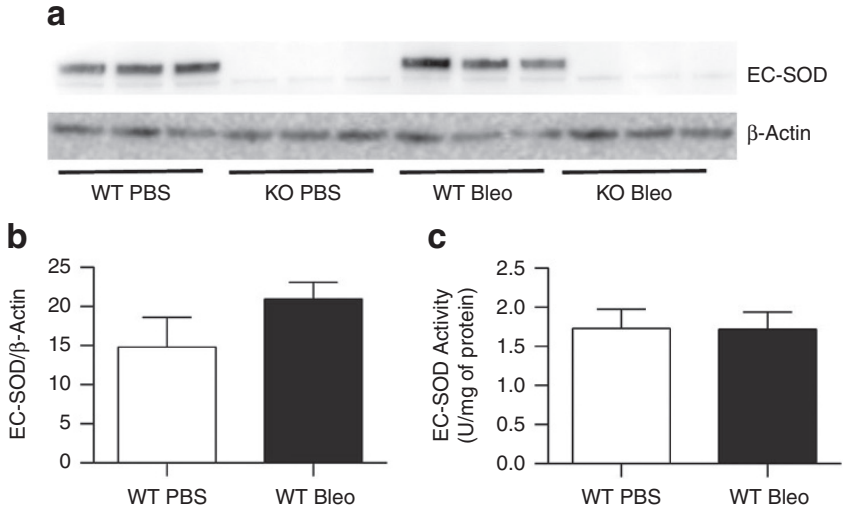

Figure 6. No change in lung EC-SOD expression or activity in WT mice treated with IP bleomycin. (a) Western blot analysis for EC-SOD and $\beta$-actin. (b) No change in EC-SOD expression in WT mice treated with bleomycin. (c) No change in EC-SOD activity in WT mice treated with bleomycin, $n=3-9$.

the changes result from the mild hypoxia in Denver ambient air. We also observed that EC-SOD KO mice developed more injury compared to WT mice in response to bleomycin. This supports the premise that a genetic predisposition or "two-hit" process can contribute to the severity of BPD.

Interestingly, it is well established that bleomycin causes lung fibrosis in adult rodents, while, consistent with our findings, two laboratories have now shown that treatment of rats with bleomycin in the neonatal period instead causes abrupted lung development $(10,14)$. A similar discrepancy is also observed in adult vs. neonatal mice in response to hyperoxia, with epithelial injury and early death in adults and, in contrast, abrupted lung development in neonates $(9,20)$. The level of EC-SOD has been shown to modulate bleomycin-induced lung fibrosis; lack of EC-SOD worsens bleomycin-induced lung fibrosis in adult mice, while overexpression of EC-SOD attenuates injury $(7,21)$. This is the first report to test the impact of decreased 


\section{Articles | Delaneyet al.}

EC-SOD expression on bleomycin-induced BPD, although we previously reported that overexpression of lung EC-SOD protected against hyperoxia-induced BPD as evidenced by decreased alveolar leukocyte counts, improved alveolar volume and surface density, and restored bronchiolar and alveolar epithelial proliferation in type II alveolar cells $(4,9)$. Since the development of PH in infants with BPD worsens clinical outcome, we next tested whether the severe alveolar injury seen with bleomycin was accompanied by $\mathrm{PH}$ and whether the $\mathrm{PH}$ was augmented by loss of EC-SOD.

The second major finding of our study is that mice lacking EC-SOD, in addition to displaying disrupted alveolar development, have evidence of $\mathrm{PH}$ at baseline, which worsens with injury. This is in contrast to the report by Xu et al. (22) that adult EC-SOD KO mice do not exhibit PH at baseline. However similar to our report, these authors did find that EC-SOD KO adult mice develop worse $\mathrm{PH}$ in response to chronic hypoxia. There are several possible explanations for the discrepancy seen in $\mathrm{PH}$ at baseline in the EC-SOD KO mice between our studies. The neonatal mice in our study were raised at Denver altitude, they were younger, and included both genders, each of which could have impacted baseline pulmonary artery pressures. We initially evaluated $\mathrm{PH}$ at $4 \mathrm{wk}$ of age, at the same time point in which we had demonstrated abrupted alveolar development. At 4 wk, we did not find evidence for bleomycin-induced $\mathrm{PH}$ by RVH or right ventricular systolic pressures, unlike the studies using the neonatal rat model of bleomycin that demonstrated BPD associated with $\mathrm{PH}$, shown by pulmonary artery pulse wave Doppler measurements and RVH $(10,14)$. We considered two important factors that could have accounted for these differences; our dosing strategy and the potential impact of bleomycin on cardiac function. We adapted the published rat model to use a similar cumulative dose of bleomycin for body weight in the mice, but to deliver it over a longer period of time, with a $1 \mathrm{wk}$ period of rest to allow for sufficient growth for the planned studies. Bleomycin-induced fibrosis in adult mice resolves with time, therefore, we speculated that the pulmonary hypertension may have also begun to resolve during that $1 \mathrm{wk}$ rest period, so we repeated the $\mathrm{RV} / \mathrm{LV}+\mathrm{S}$ measurements at $3 \mathrm{wk}$ upon completion of the bleomycin treatments. In support of this idea, bleomycin did cause RVH, which was more severe in the mice lacking EC-SOD. It is also possible that bleomycin caused a decrease in cardiac function, which could lead to lower pulmonary artery pressures despite elevated pulmonary vascular resistance. Right ventricular systolic pressure measurements do not provide information on either vascular resistance or cardiac output, though the LV hypertrophy observed in the 4-wk-old mice suggests that cardiac dysfunction was present in the neonatal mouse treated with bleomycin. Infants with BPD may develop a spectrum of pulmonary vascular disease that may not lead to echocardiographic evidence of $\mathrm{PH}$, yet likely contributes to their morbidity and survival (1). Thus in addition to testing established measures of $\mathrm{PH}$, we also examined vessel density and muscularization of small vessels in the lung. We found that vessel density is decreased and that the portion of muscularized small vessels is increased in EC-SOD KO mice at baseline compared to WT mice. While bleomycin decreases vessel density in both WT and EC-SOD $\mathrm{KO}$ strains, vascular remodeling is worse in response to bleomycin in EC-SOD KO mice. This is consistent with published data showing protection against hyperoxia induced decreases in vessel density in mice overexpressing EC-SOD (4). These findings implicate insufficient EC-SOD as a mechanism contributing to pulmonary hypertension, vascular development, and vascular remodeling in neonatal BPD.

Diverse growth factors contribute to normal lung development and blood vessel formation, including fibroblast growth factor, transforming growth factor, hepatocyte growth factor, and VEGF. VEGF signaling is essential for normal alveolar and vascular growth; VEGF and VEGFR2 are decreased in infants who died with BPD, and in models of BPD and $\mathrm{PH}$, disruption of VEGF signaling contributes to pathogenesis (23-26). Therefore, we chose to investigate whether loss of EC-SOD and/ or treatment with bleomycin affected the expression of the key molecules in this pathway. We found no alterations in VEGF/ NO signaling at $4 \mathrm{wk}$ of age, following a 1-wk period of recovery, therefore chose to repeat our experiments and measure VEGF/ $\mathrm{NO}$ signaling at $3 \mathrm{wk}$. We found that the lack of EC-SOD at baseline or treatment with bleomycin decreased lung expression of active VEGFR2 though did not impact VEGF expression. These findings are consistent with other studies in which EC-SOD content impacts VEGF signaling and angiogenesis in other settings. In hindlimb ischemia, impaired angiogenesis is worsened in EC-SOD KO mice (27) and improved in mice overexpressing EC-SOD (28). Conversely, proliferative diabetic retinopathy is associated with elevated EC-SOD and VEGF levels, indicating that elevated VEGF production enhanced by high EC-SOD levels can also increase pathologic angiogenesis (29). Altered EC-SOD can impact VEGF signaling through several different mechanisms. Overexpression of lung EC-SOD in hyperoxia-induced BPD has been shown to increase VEGF, which can then increase VEGFR2 expression (4), though we do not observe low VEGF expression in this study. Oshikawa et al. (27) identified a mechanism that could account for the impaired activation of VEGFR2 in EC-SOD KO mice independent of VEGF. They showed that EC-SODdependent production of hydrogen peroxide led to oxidation and activation of two tyrosine phosphatases, PTP1 and DEP-1, which phosphorylate VEGFR2 (27). Though decreased eNOS expression or enhanced NO scavenging by excess superoxide could contribute to elevated vascular tone, this does not likely account for low VEGFR2 in this model since eNOS-derived NO has been shown to regulate VEGFR2 by decreasing VEGF expression. Our future research will interrogate specifically how loss of EC-SOD can regulate VEGFR activation in the developing lung.

In several studies, EC-SOD activity is decreased in immature lungs, or decreases in response to injury, contributing to the imbalance in superoxide production and antioxidant scavenging. In this study, bleomycin did not decrease lung EC-SOD expression or activity at the 3 -wk time point. We previously reported that in chronic hypoxia, EC-SOD increases early 
in the course, at $3 \mathrm{~d}$, despite evidence of increased oxidative stress, but then over longer periods of exposure, activity levels ( $35 \mathrm{~d}$ of hypoxia) decrease. It is possible that EC-SOD activity is decreased at other time points or that it decreases in the pulmonary artery without changes in the total lung content. Further studies are needed to establish what effects of bleomycin-induced injury are reversible by restoring or increasing EC-SOD activity. This will be important both to better understand the pathogenesis of BPD and PH as well as develop new therapeutic strategies.

We chose to use bleomycin for our model of BPD and PH instead of other established models such as hyperoxia and hypoxia because of inconsistent reports of both BPD and PH with these models and the recent studies using bleomycin as a robust model of both alveolar and vascular disruption in neonatal rats. We acknowledge two limitations of our study, which include the potent but nonspecific effects of bleomycin on inflammation and oxidative stress, and current technical limitations in measuring right ventricular systolic pressures or utilizing echocardiography in younger mice. We also recognize the complexity of the interactions, as we note discrepant findings in both a model of skin inflammation and with melanoma-associated angiogenesis in which treatment with EC-SOD blunts VEGF-mediated pathologic angiogenesis $(30,31)$. It is not clear why loss of EC-SOD impairs physiologic VEGF signaling while enhancing pathologic VEGF expression, and this remains an important area of investigation.

In summary, we report that neonatal mice lacking EC-SOD have disrupted alveolar development, pulmonary hypertension, and pulmonary vascular remodeling at baseline. In addition, we have established a novel model of experimental BPD and PH induced by neonatal systemic treatment with bleomycin. $\mathrm{PH}$ and alveolar and vascular remodeling in the EC-SOD KO mice was associated with a marked decrease in the key growth factor receptor, VEGFR2 and eNOS. These studies demonstrate a key role for EC-SOD in normal lung development and response to neonatal injury and provide new insight into pathways amenable to therapeutic intervention for this lethal lung disease.

\section{METHODS}

\section{Mouse Model}

The University of Colorado Denver Institutional Animal Care and Use Committee (IACUC) approved all animal studies. Beginning on day 2 of life, C57/BL6 wild-type mice (Jackson Laboratory, Bar Harbor, ME) or EC-SOD knock-out mice (15) were injected with intraperitoneal bleomycin (2 units per $\mathrm{kg}$ ) (APP Pharmaceuticals, Schaumburg, IL) dissolved in PBS or PBS alone three times weekly for $3 \mathrm{wk}$ (total nine injections, $10 \mu \mathrm{l}$ ). Bleomycin dose was adjusted for body weight at each injection. Mice were euthanized for tissue harvesting, at $3 \mathrm{wk}$ or recovered for $1 \mathrm{wk}$ to enable sufficient growth to perform hemodynamic measurements at $4 \mathrm{wk}$ of age. At harvest, animals were euthanized with intramuscular ketamine $(50 \mathrm{mg} / \mathrm{kg}$, Vedco, St. Joseph, MO) and xylazine $(10 \mathrm{mg} / \mathrm{kg}$, Hospira, Lake Forest, IL) followed by exsanguination.

\section{Hemodynamic Measurements and Tissue Harvesting}

Right ventricular systolic pressures were obtained by direct RV puncture via closed chest as described (16). Lungs were flash-frozen for protein isolation or inflation-fixed at $25 \mathrm{~cm} \mathrm{H}_{2} \mathrm{O}$ with $4 \%$ paraformaldehyde for paraffin embedding.

\section{Evaluation of Alveolar Structure}

Radial alveolar counts were performed on pentachrome stained sections as previously described (17). Additional morphometric measurements were performed using computer-assisted image analysis, including mean linear intercept as a measure of intra-alveolar distance $(\mu \mathrm{m})$, nodal point density $\left(\mathrm{NP} / \mathrm{mm}^{3}\right)$ as a measure of alveolar complexity, and internal lung surface area $\left(\mathrm{mm}^{2} / \mathrm{HPF}\right)(18)$. Fields with large airways or vessels were excluded from analysis. At least six images were processed per mouse.

\section{Evaluation of Right and Left Ventricular Hypertrophy}

Hearts were removed at 3 and $4 \mathrm{wk}$ of life and dissected to isolate the free wall of the right ventricle (RV) from the left ventricle (LV) and septum (S). The ratio of $R V$ weight over $L V+S$ weight $(R V / L V+S)$ was used as an index of RV hypertrophy resulting from PH. Left ventricle weight/body weight ratio (LV/BW) were analyzed as a measure of left ventricular enlargement.

\section{Immunohistochemistry}

Immunohistochemistry was performed using the mouse monoclonal $\alpha$-smooth muscle actin antibody (1:1,000, clone1 A4; Sigma, St. Louis, MO) and MOM kit with biotinylated anti-mouse secondary IgG antibody, per instructions (Vector Laboratories, Burlingame, CA), as well as rabbit anti-human/mouse Factor VIII) (1:1,000, Sigma), with biotinylated anti-rabbit secondary IgG antibody (1:100; Vector Laboratories). Slides were developed with ImmPact DAB diluent (Vector Laboratories) and counterstained with hematoxylin.

\section{Evaluation of Vascular Structure}

Vessel density was assessed by counting the number of vessels $<30$ microns staining positive for Factor VIII per high-power field (20x). Lung fields containing large vessels or airways were excluded, and greater than six fields were included per mouse. In order to quantify the number of muscularized small vessels, the number of vessels ( $<30$ microns) that stained positive for $\alpha$-smooth muscle actin were counted and divided by the number of vessels $<30$ microns that stained positive for Factor VIII. An investigator blinded to the experimental group performed the analysis.

\section{Protein Expression}

Western blot was performed on total lung homogenates prepared in lysis buffer containing protease and phosphatase inhibitors as previously described (19) using the following antibodies: rabbit polyclonal EC-SOD antibody (1:1,000, Santa Cruz Biotechnology, Santa Cruz, CA), mouse monoclonal mouse endothelial nitric oxide synthase (eNOS) antibody (1: 500, BD Biosciences, San Jose, CA), rabbit polyclonal VEGF antibody (1:500, Santa Cruz Biotechnology), rabbit monoclonal Anti-human VEGF receptor 2 and phospho-VEGF receptor 2 (1:1,000, Cell Signaling, Danvers, MA), EC-SOD (1:1,000, Santa Cruz Biotechnology) and $\beta$-actin mouse monoclonal antibody (Sigma). The species-appropriate secondary IgG antibody was used (1:10,000, Millipore, Billerica, MA).

\section{SOD Activity Assay}

Neonate lung tissues (20-30 mg) were homogenized in five volumes of ice-cold SOD assay buffer $(50 \mathrm{mmol} / \mathrm{l}$ potassium phosphate, $\mathrm{ph} 7.4$, with $0.3 \mathrm{M}$ potassium bromide $(\mathrm{KBr}), 50 \mathrm{mmol} / \mathrm{l}$ phenylmethylsulfonyl flouride, and $3 \mathrm{mmol} / \mathrm{l}$ diethylene-triaminepentaacetic acid), and centrifuged to remove cellular debris. Total SOD activity was measured in the lung homogenates and intracellular SOD was measured in lung homogenates after concavalin A (Con A) sepharose 4B bead pull down of EC-SOD. Briefly, total lung homogenates were adjusted to a protein concentration of $1 \mu \mathrm{g} / \mu \mathrm{l}$. Control sepharose $4 \mathrm{~B}$ beads and concavalin A (Con A) sepharose 4B beads (Sigma) were washed with equilibration buffer (50 mmol/1 2-[4-(2-hydroxyethyl)piperazin-1-yl] ethanesulfonic acid (HEPES), ph 7.0, with $0.25 \mathrm{M} \mathrm{NaCl}$ ) three times and excess buffer removed. Hundred microliters $(100 \mu \mathrm{g})$ of lung homogenate were added to $75 \mu \mathrm{l}$ of either control sepharose $4 \mathrm{~B}$ beads or Con A sepharose $4 \mathrm{~B}$ beads, and incubated for $30 \mathrm{~min}$ at $4{ }^{\circ} \mathrm{C}$, followed by centrifugation at $600 \mathrm{~g}$ for $5 \mathrm{~min}$. SOD activity was measured in the supernatants using a SOD Assay kit-WST (Dojindo Molecular Technologies, Rockville, MD) according to instructions, reflecting 
total SOD activity and intracellular SOD activity. The separation of EC-SOD by Con A bead incubation was confirmed by western blot analysis showing lack of EC-SOD in the intracellular SOD sample. EC-SOD activity was determined by total SOD minus intracellular SOD measurements. The standard curve was performed using bovine erythrocyte SOD1 (Sigma). The standard curve was linear between SOD1 concentrations of $0.1-5 \mathrm{U} / \mathrm{ml}$. SOD activity data were expressed as units of SOD activity per $\mathrm{mg}$ of protein.

\section{Statistical Analysis}

Data were analyzed by two-way ANOVA, or unpaired $t$-test, using Prism (GraphPad Software, La Jolla, CA). Data were expressed as mean \pm SE and significance was defined as $P<0.05$.

\section{STATEMENT OF FINANCIAL SUPPORT}

This study was supported by National Institute Health (NIH)/National Heart Lung Blood Institute (NHLBI) HL086680 Bethesda, MD (ENG), NIH/K12 HD 068372 Bethesda, MD (C.D.), and Ikaria Neonatal Fellow Research Grant, Hampton, NJ (R.H.W.). R.H.W. was supported by Ikaria Fellow Research Grant.

\section{REFERENCES}

1. Mourani PM, Abman SH. Pulmonary vascular disease in bronchopulmonary dysplasia: pulmonary hypertension and beyond. Curr Opin Pediatr 2013;25:329-37.

2. Bhat R, Salas AA, Foster C, Carlo WA, Ambalavanan N. Prospective analysis of pulmonary hypertension in extremely low birth weight infants. Pediatrics 2012;129:e682-9.

3. Khemani E, McElhinney DB, Rhein L, et al. Pulmonary artery hypertension in formerly premature infants with bronchopulmonary dysplasia: clinical features and outcomes in the surfactant era. Pediatrics 2007;120: 1260-9.

4. Ahmed MN, Suliman HB, Folz RJ, et al. Extracellular superoxide dismutase protects lung development in hyperoxia-exposed newborn mice. Am J Respir Crit Care Med 2003;167:400-5.

5. Wedgwood S, Steinhorn RH. Role of reactive oxygen species in neonatal pulmonary vascular disease. Antioxid Redox Signal 2014;21:1926-42.

6. Nozik-Grayck E, Suliman HB, Piantadosi CA. Extracellular superoxide dismutase. Int J Biochem Cell Biol 2005;37:2466-71.

7. Van Rheen Z, Fattman C, Domarski S, et al. Lung extracellular superoxide dismutase overexpression lessens bleomycin-induced pulmonary hypertension and vascular remodeling. Am J Respir Cell Mol Biol 2011;44: 500-8.

8. Nozik-Grayck E, Suliman HB, Majka S, et al. Lung EC-SOD overexpression attenuates hypoxic induction of Egr-1 and chronic hypoxic pulmonary vascular remodeling. Am J Physiol Lung Cell Mol Physiol 2008;295:L422-30.

9. Auten RL, O’Reilly MA, Oury TD, Nozik-Grayck E, Whorton MH. Transgenic extracellular superoxide dismutase protects postnatal alveolar epithelial proliferation and development during hyperoxia. Am J Physiol Lung Cell Mol Physiol 2006;290:L32-40.

10. McNamara PJ, Murthy P, Kantores C, et al. Acute vasodilator effects of Rhokinase inhibitors in neonatal rats with pulmonary hypertension unresponsive to nitric oxide. Am J Physiol Lung Cell Mol Physiol 2008;294:L205-13.

11. Sausville EA, Peisach J, Horwitz SB. A role for ferrous ion and oxygen in the degradation of DNA by bleomycin. Biochem Biophys Res Commun 1976;73:814-22.

12. Hecht SM. DNA strand scission by activated bleomycin group antibiotics. Fed Proc 1986;45:2784-91.

13. Perveen S, Patel H, Arif A, Younis S, Codipilly CN, Ahmed M. Role of ECSOD overexpression in preserving pulmonary angiogenesis inhibited by oxidative stress. PLoS One 2012;7:e51945.

14. Tourneux P, Markham N, Seedorf G, Balasubramaniam V, Abman SH. Inhaled nitric oxide improves lung structure and pulmonary hypertension in a model of bleomycin-induced bronchopulmonary dysplasia in neonatal rats. Am J Physiol Lung Cell Mol Physiol 2009;297:L1103-11.

15. Carlsson LM, Jonsson J, Edlund T, Marklund SL. Mice lacking extracellular superoxide dismutase are more sensitive to hyperoxia. Proc Natl Acad Sci U S A 1995;92:6264-8.

16. Lizotte PP, Hanford LE, Enghild JJ, Nozik-Grayck E, Giles BL, Oury TD. Developmental expression of the receptor for advanced glycation endproducts (RAGE) and its response to hyperoxia in the neonatal rat lung. BMC Dev Biol 2007;7:15.

17. Cooney TP, Thurlbeck WM. The radial alveolar count method of Emery and Mithal: a reappraisal 1-postnatal lung growth. Thorax 1982;37: 572-9.

18. Tang JR, Karumanchi SA, Seedorf G, Markham N, Abman SH. Excess soluble vascular endothelial growth factor receptor-1 in amniotic fluid impairs lung growth in rats: linking preeclampsia with bronchopulmonary dysplasia. Am J Physiol Lung Cell Mol Physiol 2012;302:L36-46.

19. Nozik-Grayck E, Woods C, Taylor JM, et al. Selective depletion of vascular EC-SOD augments chronic hypoxic pulmonary hypertension. Am J Physiol Lung Cell Mol Physiol 2014;307:L868-76.

20. Folz RJ, Abushamaa AM, Suliman HB. Extracellular superoxide dismutase in the airways of transgenic mice reduces inflammation and attenuates lung toxicity following hyperoxia. J Clin Invest 1999;103:1055-66.

21. Bowler RP, Crapo JD. Oxidative stress in airways: is there a role for extracellular superoxide dismutase? Am J Respir Crit Care Med 2002;166(12 Pt 2):S38-43.

22. Xu D, Guo H, Xu X, et al. Exacerbated pulmonary arterial hypertension and right ventricular hypertrophy in animals with loss of function of extracellular superoxide dismutase. Hypertension 2011;58:303-9.

23. Bhatt AJ, Pryhuber GS, Huyck H, Watkins RH, Metlay LA, Maniscalco WM. Disrupted pulmonary vasculature and decreased vascular endothelial growth factor, Flt-1, and TIE-2 in human infants dying with bronchopulmonary dysplasia. Am J Respir Crit Care Med 2001;164(10 Pt 1):1971-80.

24. Le Cras TD, Markham NE, Tuder RM, Voelkel NF, Abman SH. Treatment of newborn rats with a VEGF receptor inhibitor causes pulmonary hypertension and abnormal lung structure. Am J Physiol Lung Cell Mol Physiol 2002;283:L555-62.

25. Jakkula M, Le Cras TD, Gebb S, et al. Inhibition of angiogenesis decreases alveolarization in the developing rat lung. Am J Physiol Lung Cell Mol Physiol 2000;279:L600-7.

26. Muehlethaler V, Kunig AM, Seedorf G, Balasubramaniam V, Abman SH. Impaired VEGF and nitric oxide signaling after nitrofen exposure in rat fetal lung explants. Am J Physiol Lung Cell Mol Physiol 2008;294:L110-20.

27. Oshikawa J, Urao N, Kim HW, et al. Extracellular SOD-derived $\mathrm{H} 2 \mathrm{O} 2$ promotes VEGF signaling in caveolae/lipid rafts and post-ischemic angiogenesis in mice. PLoS One 2010;5:e10189.

28. Saqib A, Prasad KM, Katwal AB, et al. Adeno-associated virus serotype 9-mediated overexpression of extracellular superoxide dismutase improves recovery from surgical hind-limb ischemia in BALB/c mice. J Vasc Surg 2011;54:810-8.

29. Izuta H, Chikaraishi Y, Adachi T, et al. Extracellular SOD and VEGF are increased in vitreous bodies from proliferative diabetic retinopathy patients. Mol Vis 2009;15:2663-72.

30. Wheeler MD, Smutney OM, Samulski RJ. Secretion of extracellular superoxide dismutase from muscle transduced with recombinant adenovirus inhibits the growth of B16 melanomas in mice. Mol Cancer Res 2003;1:871-81.

31. Kim HJ, Ham SA, Kim MY, et al. PPAR $\delta$ coordinates angiotensin IIinduced senescence in vascular smooth muscle cells through PTENmediated inhibition of superoxide generation. J Biol Chem 2011;286: 44585-93. 